\title{
Analysis of movement in primary maize roots*
}

\author{
Liyana Popova ${ }^{1,2}$, Andrea Russino ${ }^{2}$, Antonio Ascrizzi ${ }^{1,2}$ \& Barbara Mazzolai ${ }^{1}$ \\ ${ }^{1}$ Center for Micro-Biorobotics@SSSA, Istituto Italiano di Tecnologia (IIT), Viale R. Piaggio 34, 56025 Pontedera, Italy; \\ e-mail: barbara.mazzolai@iit.it \\ ${ }^{2}$ The BioRobotics Institute, Scuola Superiore Sant'Anna, Viale R. Piaggio 34, 56025 Pontedera, Italy; \\ e-mail: l.popova@sssup.it
}

\begin{abstract}
Studying plant root kinematics is important for understanding certain aspects of root growth and movement, which are strictly correlated in plants. However, there is little available data on autonomous movements in plant roots, such as nutations, and the data that are available are poorly described. We investigated the autonomous movements during growth in primary maize roots by estimating the main kinematic parameters of nutations (i.e., the period of duration and amplitude) and the growth rate. The estimations of nutation parameters were performed by developing dedicated methods, which are based on the analysis of root tip displacement and tip velocity. The data relative to the tip displacements were obtained using tip tracing software developed by our team specifically for this purpose. The results confirmed that the nutational phenomenon covers the continuous range of periods and amplitudes, with certain dominant period-amplitude types, which we clustered into three groups: i) amplitudes less than $0.1 \mathrm{~mm}$ and $4-16$ min periods, ii) amplitudes less than $0.1 \mathrm{~mm}$ and 20-120 min periods, and iii) amplitudes greater than $0.1 \mathrm{~mm}$ and $24-120 \mathrm{~min}$ periods.
\end{abstract}

Key words: maize; primary root; movement; nutation; tracking software

\section{Introduction}

Movement is a direct manifestation of plant growth and development because plants produce movements while growing (except for nastic movements) (Trewavas 2002; Trewavas 2005). Therefore, the root phenotypic plasticity, which is fundamental for plants to overcome environmental challenges, can be evaluated through its motion. For example, the accelerated growth due to light known as the 'escape tropism' in roots (Baluška et al. 2009), the particular motoric response of thigmotropism (Gilroy et al. 2008), and growth towards the moisture gradient (Eapen et al. 2005) are all pieces of evidence of how active adaptive capabilities emerge through movements. Apart from tropisms, there are some autonomous movements evoked by internal stimuli, such as root elongation and nutations (Migliaccio et al. 2009). The elongation is related to the growth rate, while the nutation is due to the asymmetrical growth that produces bending. Nutations are also called circumnutations $(\mathrm{CN})$ and can be pendulum, helical, and elliptical in nature. Generally, CNs are described by the amplitude of the apex deflection and by the period of movement duration or its frequency. There are two types of $\mathrm{CNs}$ in roots: long-period nutations with duration of 40-90 min per cycle and short-period nutations or micronutations characterised by duration of approximately $7-15$ min per cycle (Shabala \& Newman
1997; Walter et al. 2003; Shabala 2003). Although these movements are endogenous, their descriptive parameters (i.e., frequency and amplitude) may be affected by gravity (Brown 1993) and by mechanical (Ishikawa \& Evans 1992) and chemical stimulations (Hayashi et al. 2004).

Little is known about the causes of the CN phenomenon and its underlying mechanisms. However, nutations in roots were found to have some ecological implications, e.g., the anchorage of rise sprouts in flooded soil (Inoue et al. 1999). It has also been suggested that soil penetration by roots is facilitated by horizontal deflections of the apex (Hirota 1976; Vollsnes et al. 2010). However, little information about the numerical values of the CN descriptive parameters is available, whereas the methodology for studying the nutation kinematics is poorly defined. In general, the kinematics in roots may be estimated by tracing the root tip displacement (Yazdanbakhsh \& Fisahn 2010) because the movement is generated through the elongation of the apex area. In this work, we studied the kinematics of autonomous movements in roots, especially the nutation parameters, and we proposed a new methodology based on tracing root tips coupled with movement analysis by data processing. The movement analysis consisted of three phases: i) qualitative observation of the phenomenon by time-lapse videos; ii) study of the tip velocity; and iii) study of nutations by analysis of the tip trace and

\footnotetext{
* Presented at the International Symposium "Structure and function of roots", Sept. 5-9, 2011, Nový Smokovec, Slovakia
} 


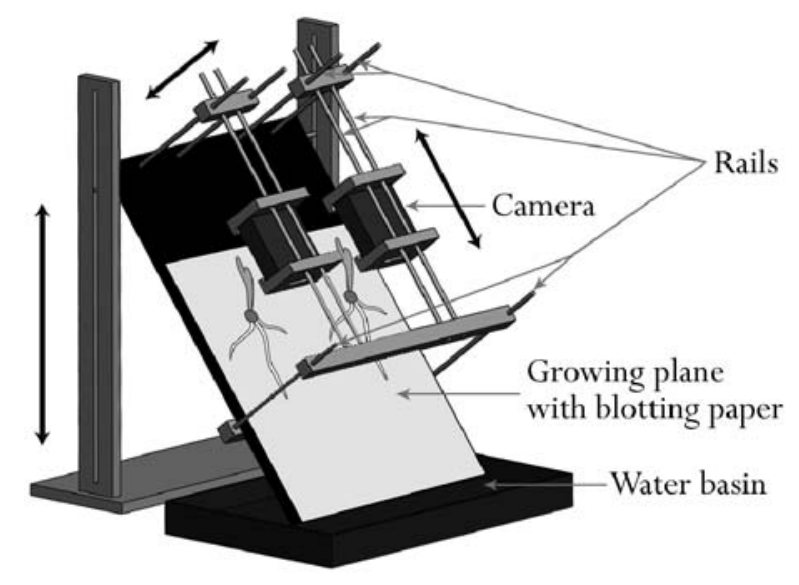

Fig. 1. The setup consists of a growing plane with blotting paper on it, a water basin to keep the plant moist, and two cameras parallel to the growing plane for time-lapse acquisitions. The inclination of the growing plane may be changed with respect to the plumb line, whereas the cameras can be positioned a desirable distance from the growing plane and from the water basin by sliding the corresponding perpendicular and parallel rails.

the tip velocity. Regarding the third point, we developed two methods for evaluating nutation parameters by analysing root tip traces. In term of CNs, we included all the nutation movements except micronutations to simplify the description of the methods. We believe that the study and understanding of autonomous movements may shed light on root growth and the associated movements.

\section{Material and methods}

\section{Setup and experimental methodology}

A simple and effective setup was built to record the growth and movements of roots in a partially constrained $2 \mathrm{D}$ space (Fig. 1). The setup consists of a plane that can slide through two side rails to manage the angle of apex penetration and two fixed cameras parallel to the growth plane.

Experiments were performed with Zea mays seeds. The experimental procedure starts with the germination of the seeds at $25^{\circ} \mathrm{C}$ in darkness for $2-3$ days in rolled germination paper $(25 \times 12 \mathrm{~cm}$, Bibby Filter Paper $)$ moistened with deionised water.

After germination, sprouts with straight-grown radicles $1-8 \mathrm{~cm}$ in length were transferred on the setup plane previously covered with a sheet of germination paper. The underside of this plane was located in a basin filled with deionised water. The whole sheet of paper is kept homogeneously wet by capillarity. If the primary roots were less than $8 \mathrm{~cm}$ after the first acquisition session, we used these sprouts again for new observations.

The experiments were conducted in a closed dark cell with a controlled temperature $\left(24-25^{\circ} \mathrm{C}\right)$ and humidity (saturated). Images were taken by cameras (Pentax OPTIO W80) with a resolution of 12 megapixels. The plants were grown in complete darkness, except for the use of the flash during the shots. The images were taken every $2 \mathrm{~min}$. This time-lapse interval was selected as an optimum trade-off between the root growth rate and the spatial resolution (i.e., at each frame, the root tip should move at least one pixel). The experimental duration ranged from $256 \mathrm{~min}$ to 1142 min.
The distance between the cameras and the growth plane of the above-described setup can be changed. In our experiments, we used a distance of $5-10 \mathrm{~cm}$, with a resulting spatial resolution of $15-26 \mu \mathrm{m} \mathrm{px}{ }^{-1}$.

\section{ARTT software}

We developed new software entitled Analyzer for Root Tip Tracks (ARTT) (Russino et al. 2011) aimed at extracting kinematic information on the movement of root tips by applying computer vision algorithms. The software elaborates the sequence of images acquired with the described setup during the root lifetime, and it traces the root tip displacements.

Tip detection is applied without the use of any markers, and tip traces are incrementally built up by linking detections over time. This process is refined through the application of a sequence of filters, which check the validity of detected tip candidates.

The software provides a graphical output of traces for an immediate test and a textual customisable output of features to be used in external statistical analyses. The extracted features are related to the tip paths and allow researchers to investigate movements in terms of kinematic changes. They include two-dimensional coordinates of tip position, displacement, velocity, movement direction, and tip orientation.

\section{Movement analysis}

We made 34 time-lapse videos of 26 sprouts to assess their root movements. We estimated the period of duration for each observed CN movement, with a maximum error of 2 shots, or $4 \mathrm{~min}$, for the detection of frames corresponding to the $\mathrm{CN}$ beginning and ending. Therefore, the maximum error of CN periods was approximately $8 \mathrm{~min}$.

We selected 14 traces with the most accurate tip detection for the numerical evaluations. These traces are all relative to roots growing with the tip kept detached from the blotting paper, as the mucilage blob generally forms when the root tip is touching paper, and the blob introduces an additional error in the tip detection by ARTT.

We selected a set of frames from each trace to compare the tip velocity with growth rate. For each set, the initial and final frames were characterised by having the root axis overlapped to cause the total tip displacement to be equal to the total root elongation. The mean growth rate was calculated as the total elongation divided by the time. The tip velocity was calculated as the Euclidean distance between two ending positions of displacement divided by its duration. The velocities were calculated for each position by 16 min sampling, yielding the average velocity to smooth fluctuations due to micronutations. The mean tip velocity was calculated from the average velocities and compared with the mean elongation rate of each track.

All 14 traces were analysed to describe the nutations. A trace was assumed to be composed of waves with different periods and amplitudes (constitutive waves). The set of these waves reflects the nutation movements encoded in the trace (Fig. 2). The aim was to find the constitutive waves that compose the trace. For this purpose, we performed the analysis of the constitutive waves by recognising them and calculating their periods and amplitudes. This analysis provided the matrix of the constitutive wave amplitudes, with columns indicating periods and rows, the tip positions in the trace. We identified the constitutive wave with the greatest amplitude in each position of the tip trace (because the wave with the greatest amplitude makes the largest contribution) for micronutations and CNs separately, as the two types of 


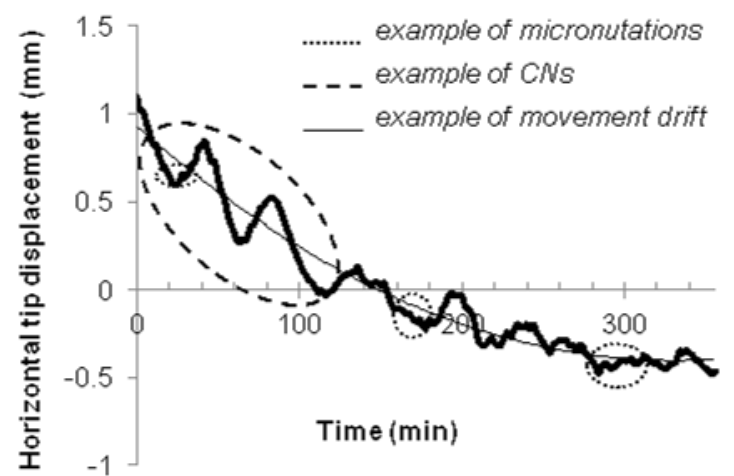

Fig. 2. A typical example of a tip trace composed of $\mathrm{CNs}$ and micronutations. In the plot, circles with dashed lines indicate the approximate positions where some of the CNs (long dash type) and micronutations (dotted dash type) are occurring, whereas a thin solid line indicates movement drift. In this example, some of the CNs and micronutations occur simultaneously.

nutation may occur simultaneously (Fig. 2). The domains of the nutation amplitudes and periods were defined by collecting these data from all 14 traces.

\section{Analysis of constitutive waves}

Different strategies may be implemented to individuate the waves from the traces. A straightforward approach may be based on first individuating the midline of the trace, or equilibrium line, and then calculating the amplitude and the period of the waves. Root tip traces result from a combination of constitutive waves that are relative to the micronutations, CNs, and drifting and that are concurrent or sometimes intermittent (Fig. 2). Thus, the resulting trace could be difficult to analyse; therefore, the midline should be individuated for each type of wave separately, considering possibly only the portions of the trace that they occur within. Therefore, a preliminary study of wave occurrence should be performed. On the contrary, the strategy adopted in this work allows us to individuate both of the wave parameters, amplitude and period, and their position in the tip trace, avoiding the preliminary occurrence study. The basic idea was to use the points of maximum amplitude, or the wave crests, for all of the calculations.

The analysis of constitutive waves of tip traces is based on two main steps: i) detection of the constitutive waves with different periods and ii) calculation of the wave amplitude. We elaborated two different methods, which differ in the first step, while the wave amplitude is calculated as half the median of the triangle formed by the corresponding three wave crests in the trace plot (Fig. 3a).

The first method is based on the elaboration of the tip displacement data, starting from the horizontal tip displacement over time during the downward growth (Fig. 3b). The wave crest corresponds to the relative minimum ( $\mathrm{min}$ ) or maximum $(\max )$ in the plot (Fig. 3b), and the distance between two consecutive minima (or maxima) is the wave period. Therefore, we found all relative minima and maxima from the horizontal tip displacement in time to individuate constitutive waves in the trace. This process was performed by sampling the trace over time, with sampling periods ranging between $2 \mathrm{~min}$ and half the trace duration (in accordance with Nyquist-Shannon sampling theorem). For each sampling, three consecutive samples of the plot were analysed. Although a max-min-max or min-max-min pattern was found, a constitutive wave with a period equal to two times the sampling period was detected (Fig. 3b).
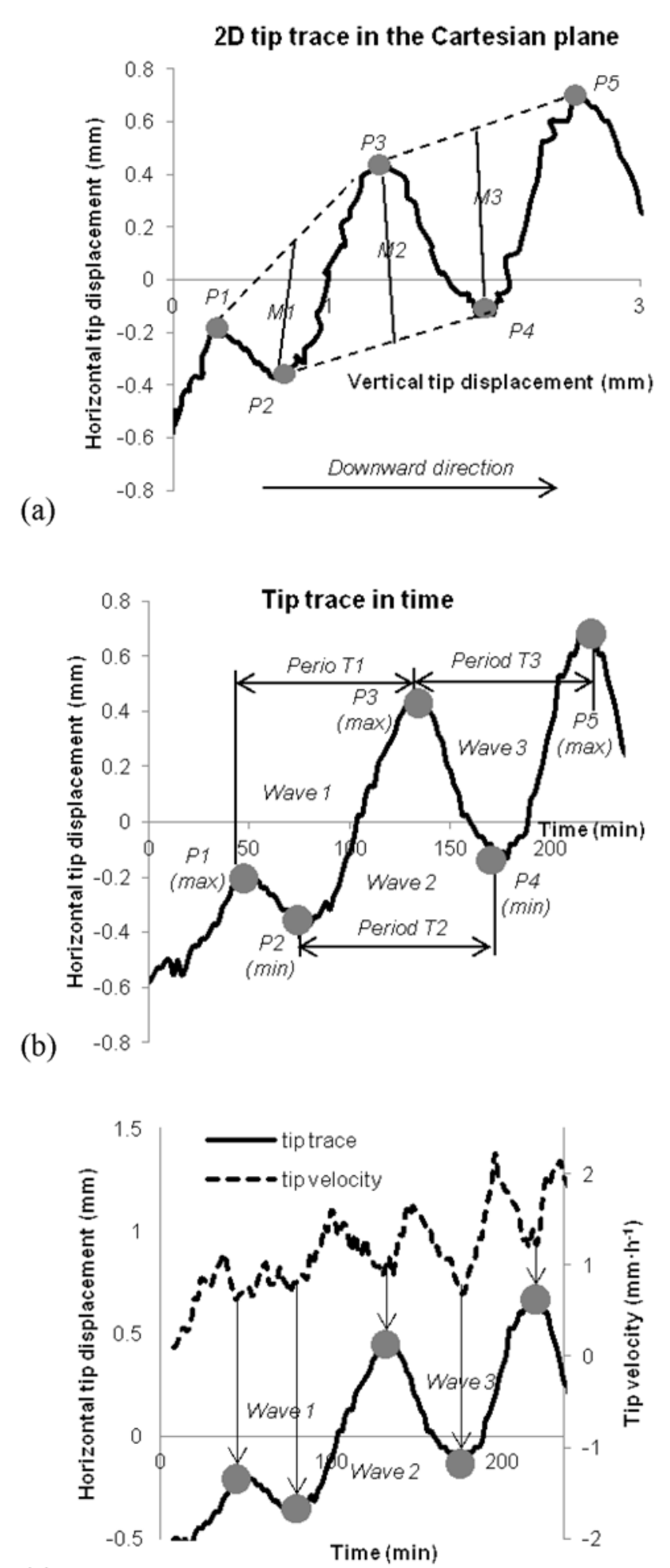

(c)

Fig. 3. The tip trace represented in different ways to detect the constitutive waves: (a) tip trace in the Cartesian plane and calculation of the constitutive wave amplitude; (b) horizontal tip displacement in time and determination of the constitutive wave period by the first method; and (c) the relationship between tip trace and tip velocity as well as the determination of the constitutive wave period by the second method.

The second method uses information obtained from the tip velocity trend. We found that a relative minimum of the tip velocity corresponds to a wave crest of the tip displace- 
ment (Fig. 3c). Therefore, we looked for the relative minima in the average velocity trends to detect the constitutive waves. The average velocities were calculated for different sampling periods ranging, as in the previous method, between $2 \mathrm{~min}$ and half the trace duration. The constitutive waves were detected by individuating three minima of the plot with the distance between the first and the third equal to four times the sampling period.

Validation of the constitutive wave analysis methods was performed by estimating the accuracy. We plotted the maximum amplitudes of the tip trace to evaluate the recognition of waves corresponding to the micronutations (416 min periods) and CNs (periods greater than $16 \mathrm{~min}$ ). Five randomly selected traces with different spatial resolutions $\left(0.015,0.016,0.0 .021,0.025\right.$, and $\left.0.026 \mathrm{~mm} \mathrm{px}^{-1}\right)$ were used for the validation. Tracks of $46 \mathrm{~min}$ were randomly selected for micronutations in these traces, whereas entire traces were used for CNs. The amplitudes and periods were calculated for the existing waves, called target waves, in each trace. Both the methods gave a set of constitutive waves distributed along the trace. For each wave in the trace, a restricted set of constitutive waves located in close proximity to its wave crest was considered. The target wave was considered to be estimated correctly if at least one wave with its amplitude and period differing by at most $20 \%$ of the target's values was found in the set of constitutive waves; otherwise, the wave was considered to be estimated incorrectly. If no constitutive wave set was found in close proximity to the target wave crest, the target wave was considered a missed recognition. The accuracy was calculated as the ratio between correctly estimated waves and the sum of all target waves.

\section{Results and discussion}

\section{Qualitative observation of time-lapse videos}

The observation of the root growth by time-lapse videos allowed us to characterise certain nutational movements. Almost all primary roots began their downward growth immediately after the sprout was positioned on the growing plane. Initially, the roots grew with the apex detached from the blotting paper, while the rest of the root was attached to the paper. After some hours, the roots grew with the tip touching the blotting paper. The root apices were observed to perform both micronutations and CNs. The CN tip trajectory assumed different shapes, such as pendulum, waving, or spiralling shapes. CNs were present occasionally or continually one after another with intermediate breaks of varying duration. We measured the periods of observed $\mathrm{CNs}$ and represented their occurrence in the histogram (Fig. 4). Because the interaction with the paper may lead to some variations in nutational parameters, we divided CNs into two groups according to the different growth conditions: i) with the apex detached from the paper on the growth plane and ii) with the apex touching the paper. Incomplete period movements, such as one-way pendulum movement, were separated from the other two groups. The CN periods covered the range of 38-110 min with the majority of the cases having 40-80 min periods.

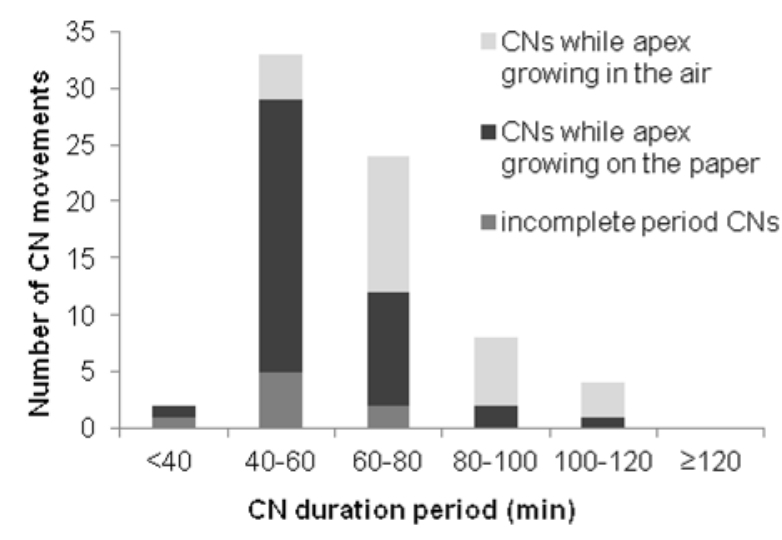

Fig. 4. Histogram of the occurrence of CN movements during the root growth. A total of 34 time-lapse videos of 26 sprouts were used for the observation of CNs. The maximum error of the $\mathrm{CN}$ duration period was approximately $8 \mathrm{~min}$ (four frames).

(a)

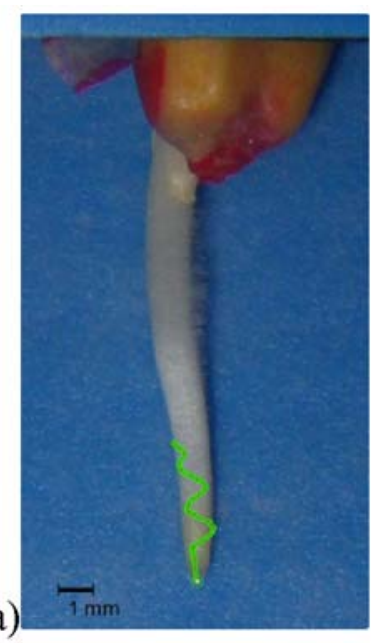

(b)

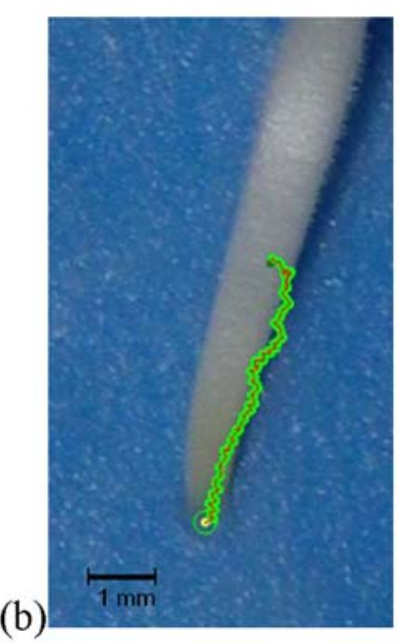

Fig. 5. Different types of movements traced by the ARTT software: (a) circumnutations and (b) micronutations.

\section{Tracing of the root tip displacements}

We elaborated the time-lapse images using the ARTT software. Non-planar movements cannot be traced by using this software because the image elaboration is performed in $2 \mathrm{D}$. However, the $2 \mathrm{D}$ projection of $3 \mathrm{D}$ movements provides a good estimation of the main nutational parameters (e.g., amplitude and period of duration) and the growth rate. ARTT traced the tip displacements of both CNs (Fig. 5a) and micronutations (Fig. 5b). We observed from the tip traces that the micronutations were present continually in some cases and eventually in others and sometimes were present together with CNs.

The study of root movements in air instead of soil introduces some artefacts because it lacks information relative to the root-soil interaction. However, observation methods for studying root movements in soil over time are still lacking. For this reason, the study of the movements outside the soil is the first step in understanding the movement modalities. 


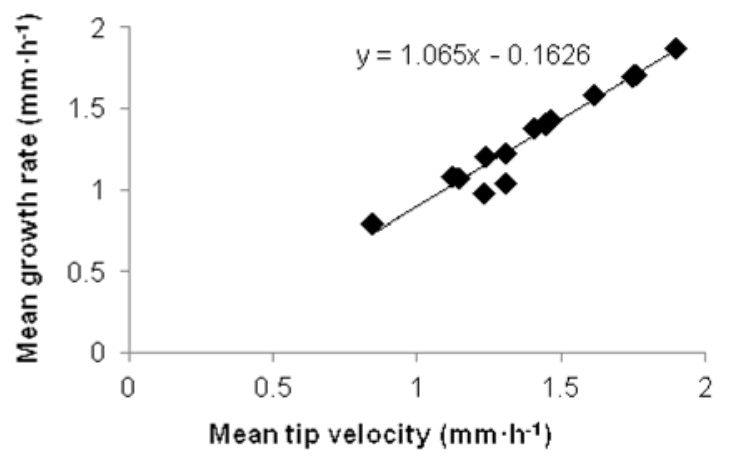

Fig. 6. Correlation between the mean tip velocity and the mean growth rate and its trendline for 14 different traces. The Pearson correlation between the tip velocities and the growth rate is approximately 0.97 . The overestimation of the growth rate by the tip velocity is approximately $6.5 \%$. The mean velocities were calculated by sampling every $16 \mathrm{~min}$.

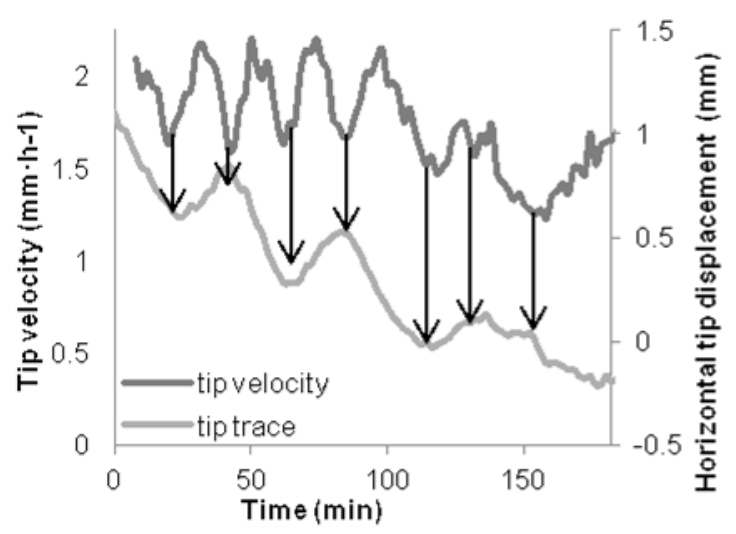

Fig. 7. Example of the relationship between the tip displacement and the tip velocity. The relative minimum of the tip velocity trend corresponds to the wave crest of the tip trace represented in time. The velocities were calculated as the vector distance of tip displacement in time. The tip velocity curves were made by sampling every $16 \mathrm{~min}$ to gather evidence of the trends while avoiding fluctuations due to the micronutations (the velocity was calculated as the vector distance between $p_{i-8}$ and $p_{i+8}$ in time $\left.\left(t_{i+8}-t_{i-8}\right)\right)$.

\section{Study of the tip velocity}

The tip displacement represents an approximation of the root growth rate. The more aligned the displacement direction of the tip and the orientation of the elongation region are, the more accurate this approximation is; otherwise, we obtain an overestimation of root growth rate. The overestimation takes place especially during the growth with $\mathrm{CNs}$, as the root apex changes its tip orientation with respect to the elongation region.

We analysed the velocity of the tip displacement to correlate it with the growth rate. The tip velocity during the interval of prevalently straight growth were calculated and compared with the mean elongation rate for all 14 cases (Fig. 6). We found that the growth rate can be estimated by the tip velocity with an overestimation of approximately $6.5 \%$.

Interestingly, we found a correlation between the

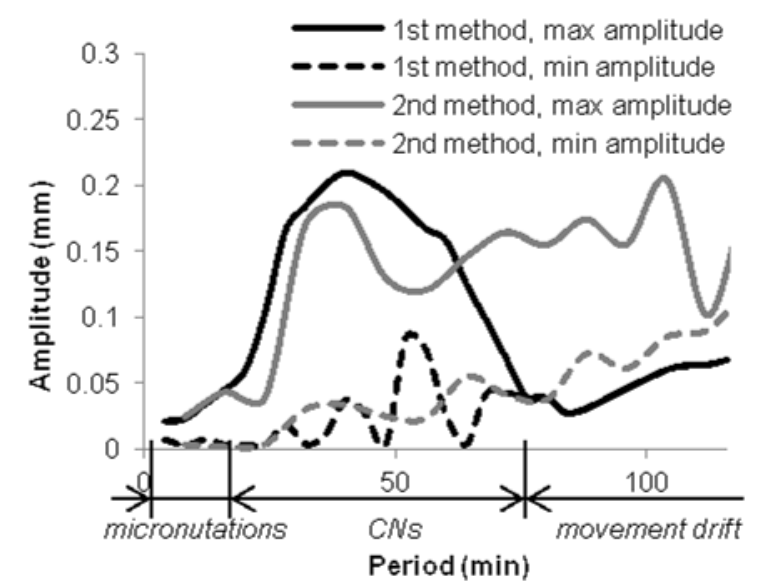

Fig. 8. Example of an amplitude-period plot with the minimum and maximum amplitudes for each period calculated by two different methods. The plot is relative to the tip trace in Fig. 2.

tip trace and the tip velocity. Indeed, the minimum values of the velocity tracks corresponded to the end point of the root horizontal deflection in the tip traces, or wave crests (Fig. 7). This result shows that the tip slows down before changing direction. We elaborated a new method for nutational movement analysis based on this finding (see methods for a more in-depth explanation). The deceleration of the tip displacement does not require the deceleration of the growth rate while the tip direction is changing with respect to the orientation of the elongation region. However, we believe that changes in the orientation of asymmetrical growth may also cause growth-rate deceleration. Further investigations are needed to verify this hypothesis.

Validation of the constitutive wave analysis methods We evaluated the functionality of both methods by estimating the accuracy of detection of micronutations and CNs. The accuracy was defined as the ratio between correctly estimated waves and the sum of correctly estimated waves, incorrectly estimated waves, and missed recognitions. We obtained an accuracy of 0.69 and 0.74 for micronutation and $\mathrm{CN}$ estimations, respectively, from the first method, and an accuracy of 0.90 for estimation of CNs with periods greater than 30 min from the second method. The accuracy of the first method is strongly dependent on the tip detection accuracy of ARTT for the micronutation detection. The second method failed in micronutation estimation and also gave inadequate results for constitutive waves with periods less than $30 \mathrm{~min}$. However, it showed a clear superiority in $\mathrm{CN}$ recognition for periods greater than $30 \mathrm{~min}$ with respect to the first method ( 0.07 rate of incorrect estimations and 0.03 rate of missed recognitions).

The two methods provided different types of constitutive wave contributions for the same trace. The first method provided an extensive set of constitutive waves for each wave of the trace because of the nonsinusoidal shape of waves in the trace. In this case, 


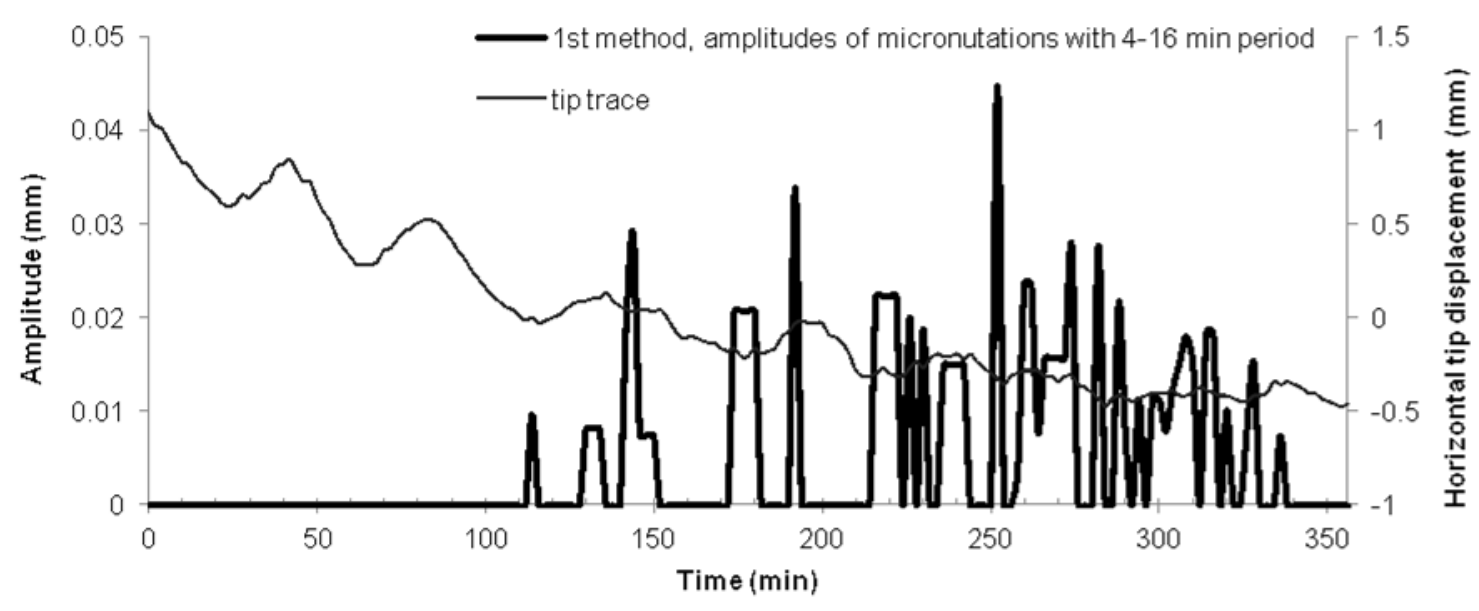

(a)

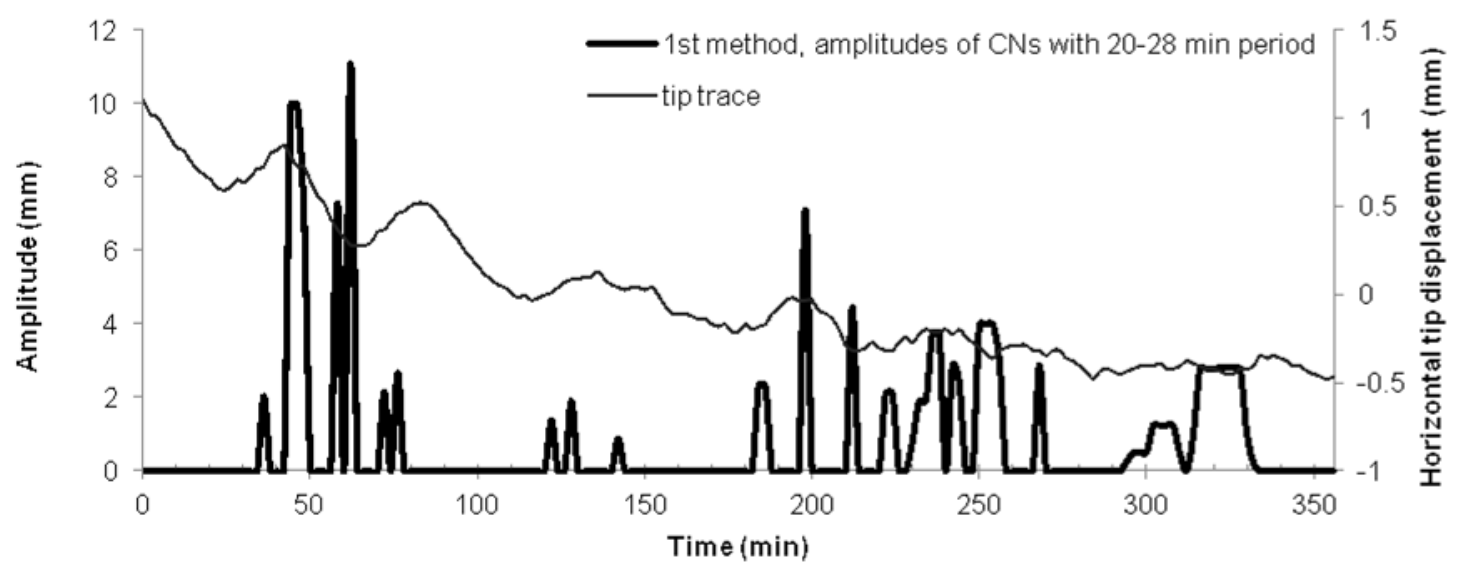

(b)

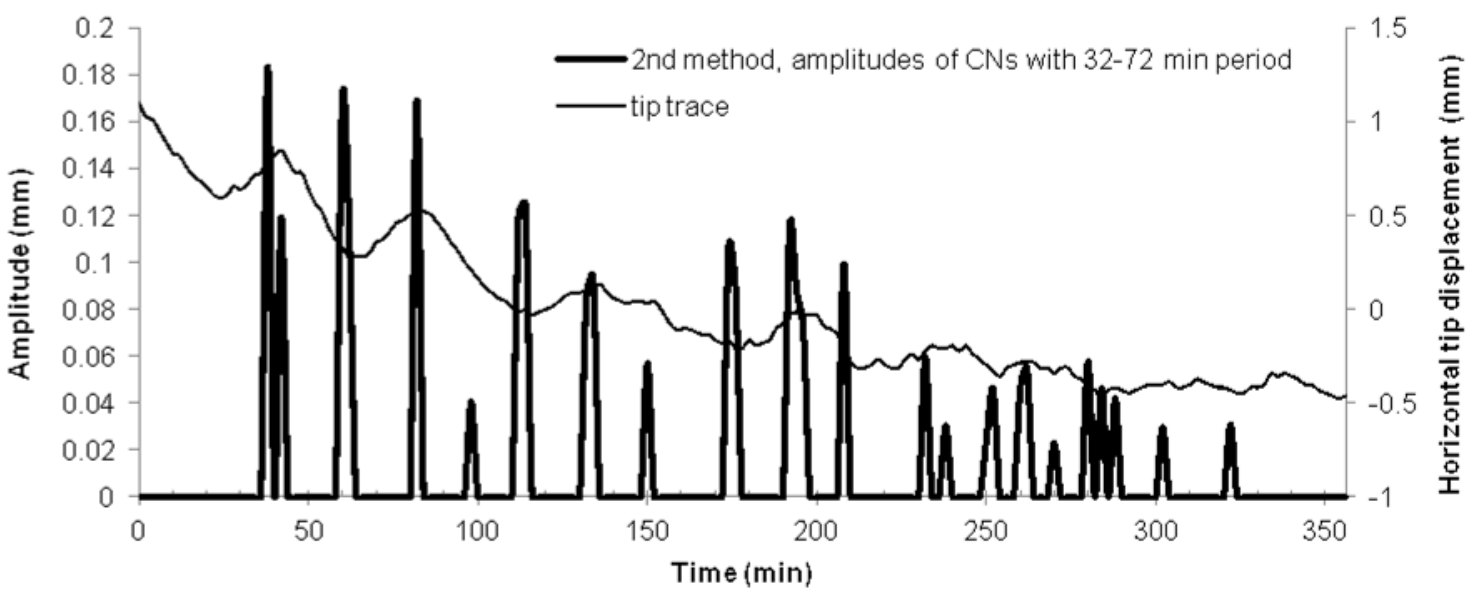

Fig. 9. Example of the representation of the constitutive waves in the tip trace: (a) amplitudes of constitutive waves relative to the micronutations calculated by the first method; (b) amplitudes of constitutive waves relative to the 20-28 min period CNs calculated by the first method; and (c) amplitudes of constitutive waves relative to the $32-72$ min period CNs calculated by the second method. The reference example is shown in Fig. 2. The amplitude picks of constitutive waves indicate the position of wave crests in the tip trace and the wave amplitude.

the constitutive wave with the greatest amplitude and period can be selected as the most representative to overcome the redundancy. The second method provided more precise results as it represented each wave of the trace with fewer constitutive waves. In this case, the multitude of constitutive waves was due to the presence of fluctuations on the wave crests of the tip trace.
Moreover, the second method provided some constitutive waves due to the inflection point of a principal wave. These inflection-point waves generally had a $32-$ 40 min period.

Both of the methods also calculated the amplitudes of the curves, which show the general trend of the tip displacement. These curves, which we called movement 
drift, are characteristic of the trace and not of the root movements. Movement drift emerges for periods greater than the CN period. The constitutive waves of movement drifts should not be taken into account in the analyses of nutational movements. To do this, the threshold of the greatest CN period should be estimated; generally, drifting waves emerge for periods greater than 120 $\min$.

Nutations can be entirely individuated and numerically described by the first method. However, the first method can miss some waves on the trace because it is strictly constrained to the direction of the root growth (the calculation should be performed with the abscissa parallel to the growth direction). In other words, if the growth direction is changing, the abscissa should change as well. In contrast, the second method is based on calculations with time as the abscissa and thus does not depend on the growth direction.

\section{Analyses of nutational movements}

The characterisation of root nutations was performed by the constitutive wave analysis consisting of two principal steps: i) constitutive wave recognition and period evaluation and ii) amplitude calculation. The amplitudes are calculated starting from the tip displacement data in both methods, whereas the first step is implemented by using different strategies. The first method also starts from the root tip displacement analysis for the constitutive wave recognition and period estimation, while the second one is based on tip velocity analysis. The minimum nutation period and the minimum difference between two nutation periods were $4 \mathrm{~min}$ for the first method and $8 \mathrm{~min}$ for the second method (two times and four times the time-lapse period, respectively). From the validation of the methods, it emerged that the first method is suitable for the description of nutations with periods less than $30 \mathrm{~min}$, whereas the second method is suitable for periods greater than 30 min.

As a first step, we explored the amplitude and period domains of the constitutive waves by applying both methods. We plotted the minimum and maximum amplitudes with respect to the wave period for each trace (one representative case is shown in Fig. 8). The minimum and maximum values of the amplitudes show the range of the horizontal tip displacements. We found that each trace presented an almost continuous spectrum of wave periods and amplitudes. The amplitudeperiod plot can be divided into three zones: i) micronutations, 8-16 min period; ii) CNs, 20-90 ( \pm 20$)$ min period; and iii) movement drift, periods greater than 90 $( \pm 20) \min ( \pm 20 \mathrm{~min}$ indicates the range of variability for the movement drift threshold).

We studied the period-amplitude distributions for micronutation and $\mathrm{CNs}$ in the traces. We plotted the maximum amplitudes along the tip trace to find waves that provide the greatest contribution. We individuated 4-28 min period waves using the first method (Fig. 9a,b). We individuated the period threshold for the movement drift for each trace (72 min for the ex-

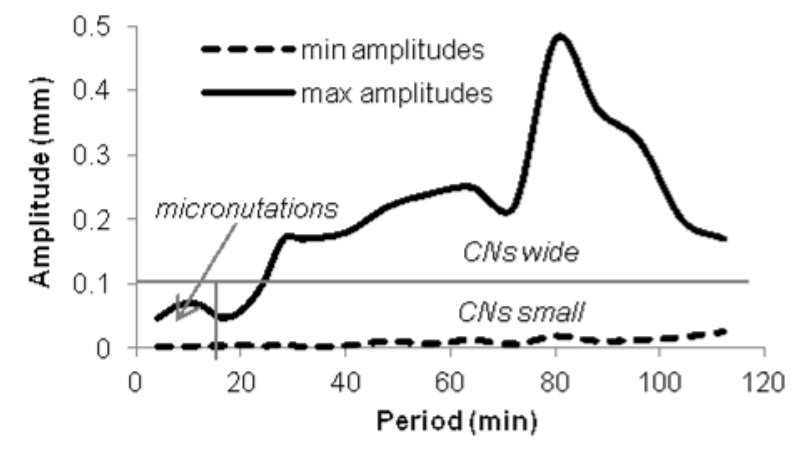

Fig. 10. Plot of the minimum ( $\min$ ) and maximum (max) amplitudes of constitutive waves represented for each period relative to the overall set of traces.

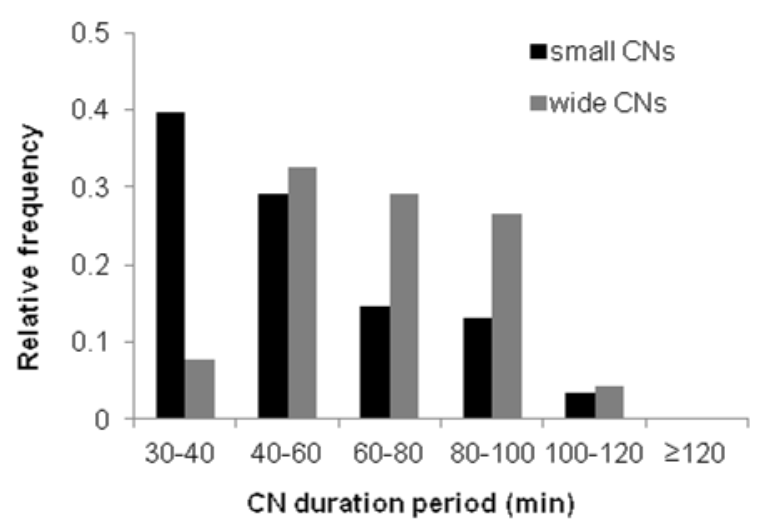

Fig. 11. Histogram of constitutive waves for small and wide CNs relative to the overall set of traces.

ample given in Fig. 9b), which is the maximum $\mathrm{CN}$ period. In a second step, the picks of $\mathrm{CN}$ waves with periods greater than $30 \mathrm{~min}$ were individuated by the second method (Fig. 9b), taking into account the previously found threshold period.

Finally, we plotted the minimum and maximum amplitudes with respect to the period of all 14 traces (Fig. 10) to describe domains of constitutive waves that reflect the domains of nutational parameters. We divided this plot into three zones by amplitude and period thresholds. The thresholds are relative to the micronutation amplitude and period, approximately $100 \mu \mathrm{m}$ and $16 \mathrm{~min}$, respectively. Interestingly, CNs may have amplitudes greater than or equal to the micronutation amplitudes. The CNs with smaller amplitudes appear to be the intermediate movements between micronutations and CNs described in the literature. Therefore, we divided the $\mathrm{CN}$ movements into two groups: i) small CNs, with amplitudes less than $0.1 \mathrm{~mm}$ and periods of $20-120 \mathrm{~min}$ and ii) wide $\mathrm{CNs}$, with amplitudes greater than $0.1 \mathrm{~mm}$ and periods of $24-120$ min. In Fig. 11, histograms of the constitutive waves for small and wide CN movements of all 14 traces are shown. The histogram of the wide CNs had a Gaussian shape with majority of the cases having 40-100 min periods ( $83 \%$ of the overall set). In contrast, the histogram of small CNs was decreasing; the majority 
of the cases had $30-60$ min periods ( $69 \%$ of the overall set).

We believe that the analysis of root kinematics may be useful for estimating the root responses to the changing biotic and/or abiotic factors. Apart from the root growth rate, the parameters of nutations are the fundamental descriptors of kinematics. The methods proposed here enable quantitative estimation of root kinematics to make the comparison of different growth conditions relevant.

\section{References}

Baluška F., Mancuso S., Volkmann D. \& Barlow P.W. 2009. The 'root-brain' hypothesis of Charles and Francis Darwin. Plant Sig. Behav. 4: 1121-1127.

Brown A.H. 1993. Circumnutations:from Darwin to space flights. Plant. Physiol. 101: 345-348.

Eapen D., Barroso M.L., Ponce G., Campos M.E. \& Cassab G.I. 2005. Hydrotropism: root growth responses to water. Trends Plant Sci. 10: 44-50.

Gilroy S., Monshausen G.B. \& Swanson S.J. 2008. Chapter 5. Touch Sensing and Thigmotropism, pp. 91-122. In: Gilroy S. \& Masson P.H. (eds), Plant Tropisms, Blackwell Publishing Ltd, Oxford, UK.

Hayashi Y., Nishiyama H., Tanoi K., Ohya T., Nihey N., Tanioka K. \& Nakanishi T.M. 2004. An alluminium influence on root circumnutation in dark revealed by a new super-HARP (highgain avalanche rushing amorphous photoconductor) camera. Plant. Cell Physiol. 45: 351-356.

Hirota H. 1976. Rotation growth of root tips in Zea mays and Lolium multiflorum. J. Jap. Sci.Grassl. Set. 22: 156-160.
Inoue N., Arase T., Hagiwara M., Amano T., Hayashi T. \& Ikeda R. 1999. Ecological significance of root tip rotation for

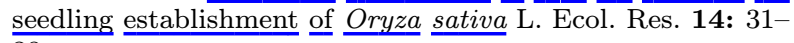
38 .

Ishikawa H. \& Evans M.L. 1992. Induction of curvature in maize roots by calcium or by thigmostimulation. Role of the postmitotic isodiametric growth zone. Plant. Physiol. 100: 762-768.

Migliaccio F., Fortunati A. \& Tassone P. 2009. Arabidopsis root growth movements and their symmetry: Progress and problems arising from recent work. Plant Sig. Behav. 4: 183-190.

Russino A., Ascrizzi A. \& Mazzolai B. 2011. A novel imageanalysis tool for study of root tip movements. Proceedings of 7 th International Symposium on Structure and Function of Roots. Nový Smokovec, Slovakia, pp. 148-149.

Shabala S.N. \& Newman I.A. 1997. Proton and calcium flux oscillations in the elongation region correlate with root nutation. Plant.Physiol. 100: 917-926.

Shabala S. 2003. Physiological implications of ultradian oscillations in plant roots. Plant Soil 255: 217-226.

Trewavas A. 2002. Mindless mastery. Nature 415: 841

Trewavas A. 2005. Plant intelligence. Naturwissenschaften 92: 401-413

Vollsnes A. V., Futsaether C.M.\& Bengough A.G. 2010. Quantifying rhizosphere particle movement around mutant maize roots using time-lapse imaging and particle image velocimetry. Soil Sci. 61: 926-939.

Walter A., Feil R. \& Schurr U. 2003. Expansion dynamics, metabolite composition and substance transfer of the primary root growth of Zea mays L. growth in different external nutrient availabilities. Plant. Cell. Environ. 26: 1451-1466.

Yazdanbakhsh N. \& Fisahn J. 2010. Analysis of Arabidopsis thaliana root growth kinetics with high temporal and spatial resolution. Ann. Bot. 105: 783-791.

Received November 16, 2011 Accepted January 27, 2012 